\title{
Template-Directed Synthesis of Silica Nanotubes for Explosive Detection
}

\author{
Adem Yildirim, ${ }^{+, \neq}$Handan Acar, ${ }^{+, \neq}$Turan S. Erkal, $^{+, \neq}$Mehmet Bayindir, ${ }^{*,+,, \S}$ and Mustafa O. Guler ${ }^{*,+, \neq}$ \\ ${ }^{\dagger}$ Institute of Materials Science and Nanotechnology, ${ }^{\ddagger}$ National Nanotechnology Research Center (UNAM), and ${ }^{\S}$ Department of \\ Physics, Bilkent University, 06800 Ankara, Turkey
}

Supporting Information

ABSTRACT: Fluorescent porous organic-inorganic thin films are of interest of explosive detection because of their vapor phase fluorescence quenching property. In this work, we synthesized fluorescent silica nanotubes using a biomineralization process through self-assembled peptidic nanostructures. We designed and synthesized an amyloid-like peptide selfassembling into nanofibers to be used as a template for silica nanotube formation. The amine groups on the peptide nanofibrous system were used for nucleation of silica nanostructures. Silica nanotubes were used to prepare highly porous surfaces, and they were doped with a fluorescent dye by physical adsorption for explosive sensing. These porous surfaces exhibited fast, sensitive, and highly selective fluorescence quenching against nitro-explosive vapors. The materials developed in this work have vast potential in sensing applications due to enhanced surface area.

KEYWORDS: biomimetic, peptide, template-directed synthesis, self-assembly, silica, explosive detection

\section{INTRODUCTION}

Inorganic nanotubes have been attracting a great deal of interest due to their potential applications in sensing, catalysis, and biotechnology. ${ }^{1,2}$ Silica is one of the most studied materials to produce nanotubes because of its inert nature, hydrophilicity, and ease of surface functionalization. ${ }^{3}$ Silica nanotubes are typically prepared within the pores of anodic alumina membranes by chemical vapor deposition or sol-gel methods. ${ }^{4-6}$ Wall thickness and pore size of the tubes can be controlled by changing the reaction conditions. However, the size of the tubes is restricted with the pore sizes of the membranes, and it is not easy to produce tubes thinner than $50 \mathrm{~nm}$. Alternatively, silica nanotubes were prepared around the organic or inorganic templates such as carbon nanotubes, ${ }^{7,8} \mathrm{ZnO},{ }^{9}$ and $\mathrm{PbSe}^{10}$ nanowires, gold nanorods, ${ }^{11}$ surfactants, ${ }^{12,13}$ peptides, ${ }^{14}$ and gels. ${ }^{15,16}$ Depending on the template used, a variety of nanotubes with different sizes, pore diameters, and wall thicknesses were obtained. However, large scale production of thin $(<50 \mathrm{~nm})$ and long $(<1 \mu \mathrm{m})$ silica nanotubes is still rare. ${ }^{17}$ Therefore, high aspect ratio silica nanotubes are indispensible in catalysis and sensing applications because of their high surface area and ability to form highly porous networks.

Recently, we developed thin and long silica nanotubes using amyloid-like peptide (ALP) nanofibers as template. ${ }^{18}$ The peptide nanofibers not only act as a template but also catalyze the silica formation because of the amine groups on their periphery. We designed and synthesized a short peptide sequence, Ac-KFFAAK-
Am (Figure S1a, Supporting Information), which forms nanofibrous structures through hydrogen bonding directed self-assembly. The amine groups of the lysine residues in the peptide sequence serve as nucleation centers for silica precursors. Removal of the organic template after silica formation can be achieved by a calcination process at an appropriate temperature or by washing with excess acidic methanol solution. The silica nanotubes have small pores $(2-3 \mathrm{~nm})$, and their wall thicknesses can be tuned between 10 and $25 \mathrm{~nm}$. The facile preparation process enables large scale production of high aspect ratio silica nanotubes (Scheme 1).

Fluorescent quenching based sensing methods are promising for rapid and sensitive detection of explosive vapors. ${ }^{19}$ The mechanism of fluorescence quenching is based on photoinduced electron transfer from excited fluorescent molecules to analytes. ${ }^{20}$ Previously, conjugated polymers, ${ }^{19-23}$ fluorescent organic nanofibrils, ${ }^{24,25}$ dye doped porous silica films, ${ }^{26-29}$ and dye decorated silica nanoparticles ${ }^{30}$ were used for fluorescence quenching based sensing of explosives. In this work, we utilized mesotetrakis-p-carboxy-phenylporphyrin $\left(\mathrm{TCPPH}_{2}\right)$ doped silica nanotubes that were synthesized through peptide nanostructure templating for explosive sensing. The fluorescent silica nanotubes (FSNTs) can be dispersed in ethanol or in water and can be directly casted on the surface to form a porous network. The

\section{Received: August 2, 2011}

Accepted: September 26, 2011

Published: September 26, 2011 
Scheme 1. Schematic Illustration of Preparation of Silica Nanotubes and Porous Fluorescent Silica Nanotube Sensor Surface

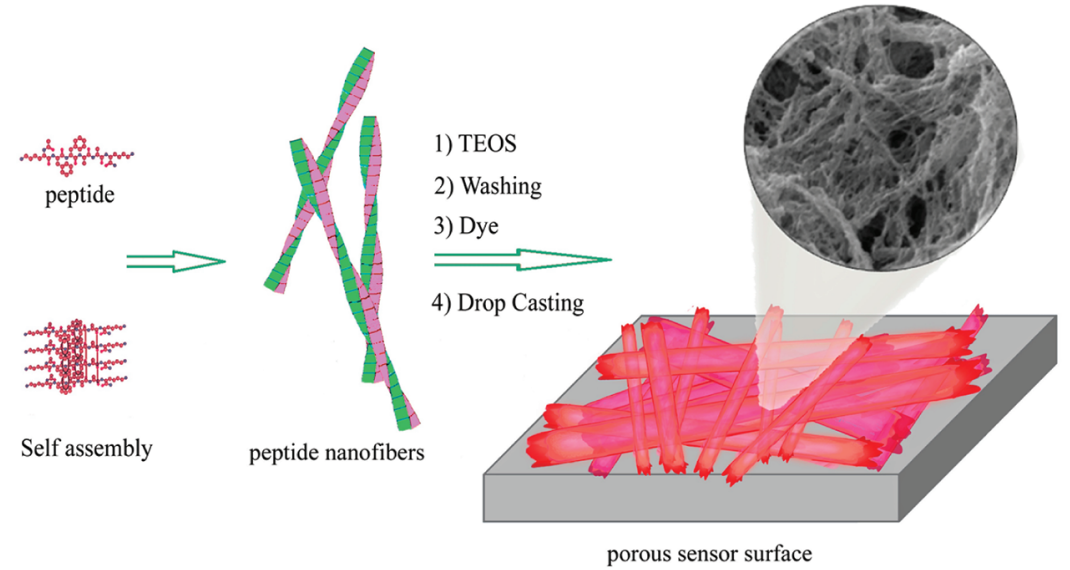

porous FSNT surface provides sensitive detection of nitroaromatic explosives: trinitrotoluene (TNT) and dinitrotoluene $(\mathrm{DNT}) .^{26-28}$ In order to demonstrate the effect of silica nanotube surface area on sensor performance, we also analyzed $\mathrm{TCPPH}_{2}$ doped nonporous silica thin film and silica nanoparticle sensors. Furthermore, the selectivity of the FSNT sensor is tested with vapors of other aromatic molecules including nitrobenzene (NB), toluene, xylene, benzoic acid (BA), and dihydroxybenzoic acid (DBA).

\section{EXPERIMENTAL SECTION}

2.1. Materials. Fmoc and Boc protected amino acids, MBHA Rink Amide resin, and HBTU were purchased from NovaBiochem and $\mathrm{ABCR}$. Meso-tetrakis-p-carboxy-phenylporphyrin $\left(\mathrm{TCPPH}_{2}\right)$ was purchased from Frontier Scientific (USA). The other chemicals were purchased from Fisher, Merck, Alfa Aesar, or Aldrich and used as provided.

2.2. Peptide Synthesis. Peptides were constructed on MBHA Rink Amide resin. Amino acid coupling reactions were performed with 2 equivalents of Fmoc protected amino acid, 1.95 equivalents of HBTU, and 3 equivalents of DIEA for $2 \mathrm{~h}$. Fmoc protecting group removal was performed with $20 \%$ piperidine/DMF solution for $20 \mathrm{~min}$. Cleavage of the peptides from the resin was carried out with a mixture of TFA/TIS/ $\mathrm{H}_{2} \mathrm{O}$ in ratio of 95:2.5:2.5 for $3 \mathrm{~h}$. Excess TFA was removed by rotary evaporation. The remaining peptide was triturated with ice-cold diethyl ether, and the resulting white precipitate was freeze-dried. The peptide was characterized by liquid chromatography and quadruple-time-offlight mass spectrometry (Q-TOF MS) equipped with an ESI source (Figure S1b, Supporting Information). The mass spectrum shows the corresponding mass of the peptide, and the purity of the peptide was assessed by RP-HPLC and found to be more than 95\% (Figure S1c, Supporting Information).

2.3. Self-Assembled Template Formation, Silica Mineralization, and Silica Nanotube Preparation. Silica mineralization was achieved using tetraethyl orthosilicate (TEOS) as a precursor. TEOS $(20 \mu \mathrm{L})$ was added on to the peptide gel $(10 \mathrm{mg}$ in $1 \mathrm{~mL}$ of ethanol), and the sample was aged for 1 week. Then, samples were washed with an excess amount of acidic methanol $(10 \mu \mathrm{L}$ of $1 \mathrm{M} \mathrm{HCl}$ per $1 \mathrm{~mL}$ of methanol). Five $\mathrm{mL}$ of acidic methanol was added into the silica coated peptide fiber gels and vortexed for $3 \mathrm{~min}$. Later, the solutions were centrifuged at $10000 \mathrm{rpm}$ for $5 \mathrm{~min}$. Supernatant was removed, and fresh acidic methanol was added. This procedure was repeated for 5 times. At the last repeat after vortexing, the solution was not centrifuged and the sample for TEM imaging was obtained from this solution.
2.4. Fluorescent Silica Nanotube (FSNT) Sensor Surface Preparation. After the organic template removal, silica nanotubes were dispersed in $1 \mathrm{~mL}$ of ethanol and $20 \mu \mathrm{g}$ of $\mathrm{TCPPH}_{2}$ was added in $100 \mu \mathrm{L}$ of ethanol. The solution was incubated 1 day at room temperature. Then, the solution was diluted with ethanol (1:50). Finally, $100 \mu \mathrm{L}$ portions of the diluted solution was coated on glass substrates by drop casting and dried in an oven at $60{ }^{\circ} \mathrm{C}$. The glass substrates were sonicated in ethanol before coating.

2.5. Sensor Surface Preparation with Nonporous Silica Thin Film. A nanoporous silica thin film was prepared according to a previous report. $^{26}$ TEOS $(2.25 \mathrm{~mL}), 2.05 \mathrm{~mL}$ of ethanol, $0.1 \mathrm{mg}$ of $\mathrm{TCPPH}_{2}$ in $0.2 \mathrm{~mL}$ of ethanol, $0.2 \mathrm{~mL}$ of water, and $5 \mu \mathrm{L}$ of $\mathrm{HCl}(0.1 \mathrm{M})$ were mixed and stirred at $60^{\circ} \mathrm{C}$ for $90 \mathrm{~min}$; then, $0.4 \mathrm{~mL}$ of $\mathrm{HCl}(0.1 \mathrm{M})$ and $0.35 \mathrm{~mL}$ of water were added and the solution was further stirred for $15 \mathrm{~min}$ at room temperature. Finally, the sol was aged at $50{ }^{\circ} \mathrm{C}$ for $15 \mathrm{~min}$. Three $\mathrm{mL}$ of aged sol was diluted with $3 \mathrm{~mL}$ of ethanol and coated on glass substrates with spin-coating at $2000 \mathrm{rpm}$.

2.6. Sensor Surface Preparation with Silica Nanoparticles. Silica nanoparticles with diameters around $60 \mathrm{~nm}$ were prepared according to Stöber method. ${ }^{31}$ Briefly, $0.25 \mathrm{~mL}$ of water, $10 \mathrm{~mL}$ of ethanol, and $2.56 \mathrm{~mL}$ of ammonia solution (26\%) were mixed with $1.5 \mathrm{~mL}$ of TEOS in $40 \mathrm{~mL}$ of ethanol. The resulting solution was stirred at $25^{\circ} \mathrm{C}$ for $24 \mathrm{~h}$. The particles were collected by centrifugation ( $9000 \mathrm{rpm}$ for $30 \mathrm{~min}$ ), washed several times with water and ethanol, and dried in a vacuum oven. Twenty $\mathrm{mg}$ of particles dispersed in $10 \mathrm{~mL}$ of ethanol and $0.2 \mathrm{mg}$ of $\mathrm{TCPPH}_{2}$ was added in $0.2 \mathrm{~mL}$ of ethanol and stirred overnight. The particles were collected by centrifugation $(9000 \mathrm{rpm}, 30 \mathrm{~min}$ ) and dispersed in $10 \mathrm{~mL}$ of ethanol. The dye doped silica nanoparticle dispersion $(0.1 \mathrm{~mL})$ was diluted with $0.9 \mathrm{~mL}$ of ethanol, and $75 \mu \mathrm{L}$ portions of the dispersion were drop casted on clean glass substrates. The substrates were dried in an oven at $40{ }^{\circ} \mathrm{C}$.

2.7. Fluorescence Quenching Experiments. The fluorescence quenching experiments were performed similar to the previous reports. $^{26-28}$ Approximately $0.1 \mathrm{~g}$ of analytes were placed into a $15 \mathrm{~mL}$ vial and covered with cotton to prevent direct contact of the analyte molecules with the films and to maintain constant analyte pressure. For nitrobenzene, toluene, and xylene, which are liquid at $\mathrm{RT}$, a small droplet was placed into a vial and covered with cotton. The FSNT surfaces were exposed to the analyte vapors for a specified period of time, and fluorescence measurements were carried out immediately. Measurements were performed at room temperature, and it is assumed that the vial atmosphere was saturated with analyte vapor. ${ }^{26}$

2.8. Characterization. Liquid Chromatography/Mass Spectrometry (LC/MS). LC/MS measurements were performed on Agilent Technologies 1200/6530 Accurate-Mass Q-TOF LC/MS. Agilent Zorbax 

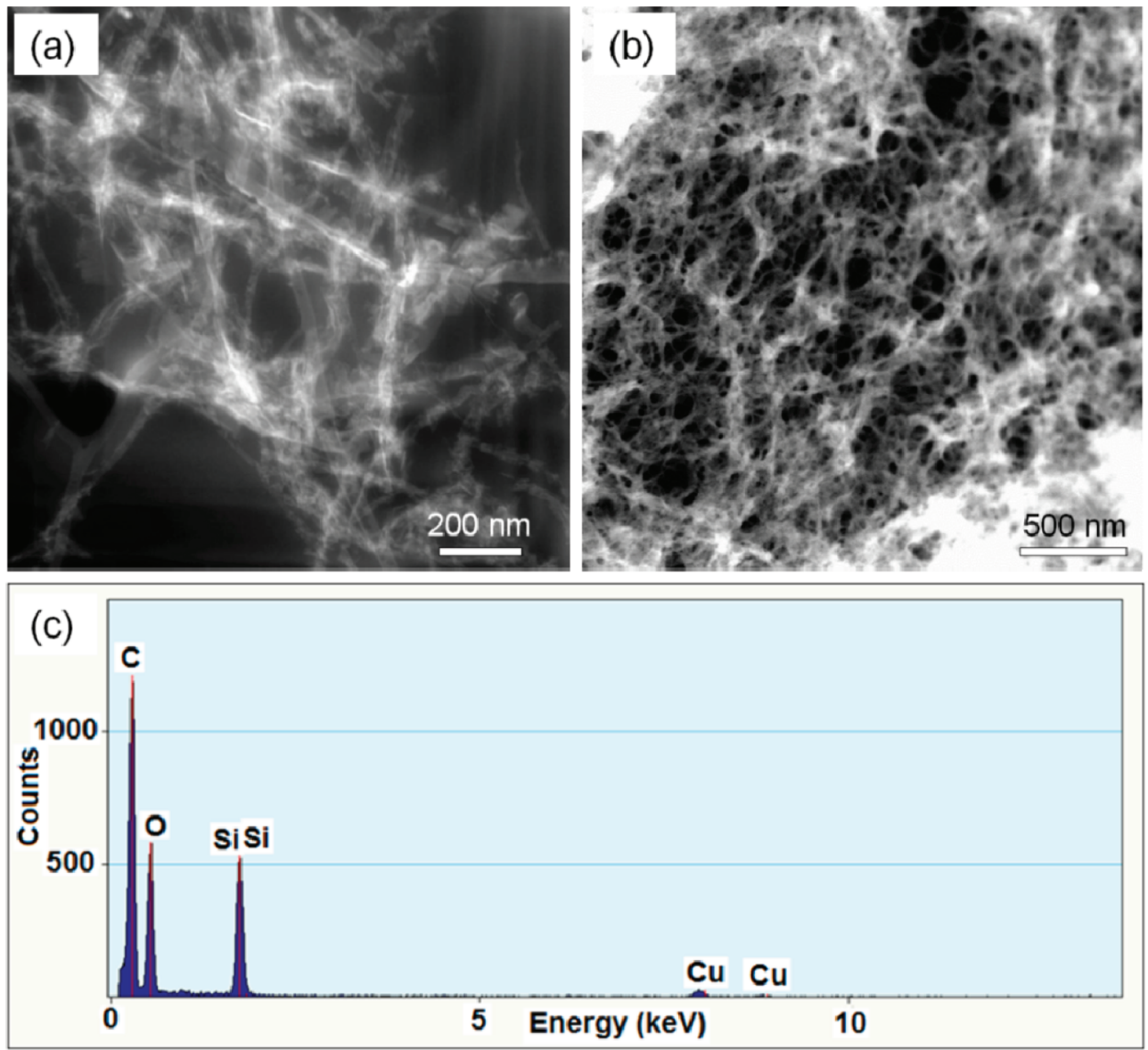

Figure 1. ( $a$ and b) STEM images revealed silica nanotube formation. (c) EDX spectrum of the silica nanotubes after template removal did not show signals related to peptide template.

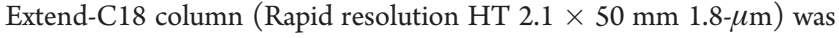
used with a gradient of $(\mathrm{A})$ water $(0.1 \%$ formic acid $)$ and $(\mathrm{B})$ acetonitrile (0.1\% formic acid).

Scanning Electron Microscopy (SEM). A FEI Nova 600i Nanolab scanning electron microscope was used. Calcinated or acidic methanol washed silica nanotubes on silicon wafers were used for SEM analysis.

Transmission Electron Microscopy (TEM) and Scanning Transmission Electron Microscopy (STEM). TEM and STEM were performed with FEI Tecnai G2 F30. Diluted samples were placed on a Lacey mesh ultrathin carbon coated copper grid. Uranyl acetate solution ( $2 \%(\mathrm{wt} / \mathrm{v}))$ was used for staining organic nanostructures. Ten $\mu \mathrm{L}$ of diluted sample was dropped on a grid for $1 \mathrm{~min}$. The excess amount was removed by pipetting. Then, $2 \%$ uranyl acetate solution was put on a parafilm sheet. The grid was put on the drop upside down for $5 \mathrm{~min}$. The grids were dried in the fume hood at room temperature overnight. Silica samples were directly placed onto the TEM grid.

Atomic Force Microscopy. AFM images of the sensor surfaces were recorded using MFP-30 Asylum Research AFM operated in tapping mode at a frequency of $246 \mathrm{kHz}$. AFM images were taken at $512 \times 512$ pixels resolution. Image was taken with spring constant $40 \mathrm{~N} / \mathrm{m}$, and the set point and scanning speed were $0.7-1.0 \mathrm{~V}$ and $1.0-1.5 \mathrm{~Hz}$, respectively.

UV-Vis Absorption and Fluorescence Spectroscopies. UV-vis absorption spectra were recorded by a Varian Carry100 spectrophotometer. Fluorescence spectra were recorded by a Varian Eclipse spectrophotometer.

Spectroscopic Ellipsometry. Thickness of the nonporous silica film was determined by a J. A. Woollam V-Vase Ellipsometer in reflection mode.

\section{RESULTS AND DISCUSSION}

We designed and synthesized the ALP molecule (Ac-KFFAAKAm) to mimic the amyloid proteins composed of extended sheetlike peptide secondary structures. The ALP molecules formed extended hydrogen-bonded supramolecular structures. Relatively strong intermolecular interactions result in stable assemblies. Two phenyl groups of the ALP molecule promote $\beta$-sheet formation ${ }^{32}$ through $\pi-\pi$ stacking. ${ }^{33}$ The amine groups on the side chains of the lysine residues act as nucleation sites for silica precursors and catalyze the polymerization reaction of silica precursor due to their basic nature. The ALP molecules form a gel in ethanol at a minimum concentration of $1 \mathrm{mg} / \mathrm{mL}$. On the other hand, ALPs readily dissolve in methanol and in acidic water. In this study, we prepared ALP gels in ethanol and the ALP concentration was $10 \mathrm{mg} / \mathrm{mL}$. ALP gels formed a highly porous 3-D network consisting of nanosized fibers. The nanofibrous structure of the ALP gel was also investigated with TEM (Figure S2, Supporting Information). According to Figure S2, Supporting Information, the diameter of the ALP nanofibers was about $15-20 \mathrm{~nm}$ and the length was in the micrometer range.

The ALP nanofibers in ethanol were used as templates for silica nanotube growth. Silica mineralization was achieved using tetraethyl orthosilicate (TEOS) as a precursor. TEOS $(20 \mu \mathrm{L})$ was added into the gel $(1 \mathrm{~mL})$, and the sample was aged for 1 week. After silica polymerization, the gels were dissolved with excess acidic methanol to remove the ALP molecules for 5 times. 


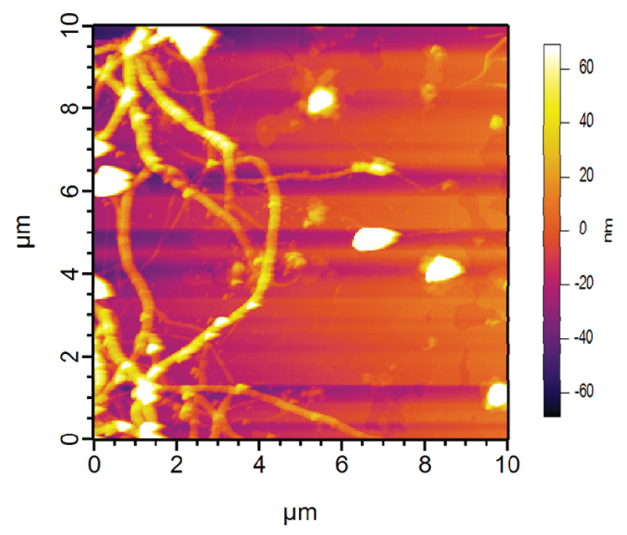

Figure 2. AFM image of the fluorescent silica nanotube sensor surface.

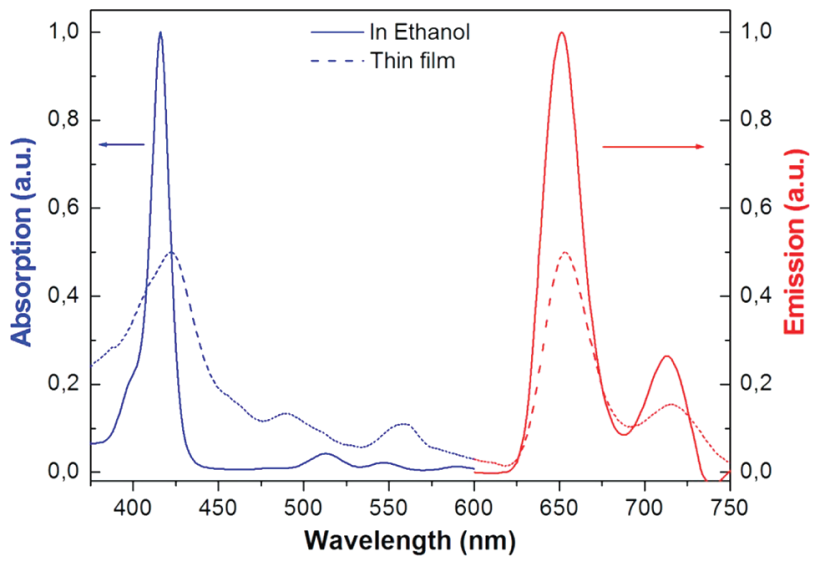

Figure 3. Absorption (blue) and emission (red) spectra of $\mathrm{TCPPH}_{2}$ in ethanol (dashed line) and in silica nanotubes (solid line).

Silica nanotubes (SNTs) were dispersed in methanol. Then, $\mathrm{TCPPH}_{2}$ dye was added, and the dispersion was aged for 1 day for physisorption of the dye to the silica network. The SNTs were observed by TEM (Figure 1a,b) and SEM (Figure S3, Supporting Information) after template removal. Both SEM and TEM images revealed formation of high aspect ratio silica nanotubes with thickness around $30 \mathrm{~nm}$. The nitrogen atom was not observed in the EDX spectrum of the region observed on TEM image (Figure 1c), which is an evidence of the removal of the peptide molecules. The carbon and copper signals were caused by the TEM grid. Nitrogen and carbon signals were also not observed on the EDX spectrum of the SEM image shown in Figure S3c, Supporting Information.

Sensor surfaces were prepared by drop casting of FSNT dispersions on glass substrates. The FSNT sensor surface was characterized with atomic force microscopy (AFM). The AFM image indicated individual nanotubes as well as thicker structures formed by aggregation of nanotubes (bundles) (Figure 2). Randomly oriented FSNTs formed a highly porous network. The absorption and emission spectra of FSNTs on a glass surface are shown in Figure 3. Identical emission spectra of $\mathrm{TCPPH}_{2}$ in ethanol and in SNTs confirm incorporation of dye molecules in the silica network.

In order to test selectivity and sensitivity of the FSNT sensor, vapor phase fluorescence quenching experiments were performed with TNT, DNT, and five other similar chemicals (NB, toluene,
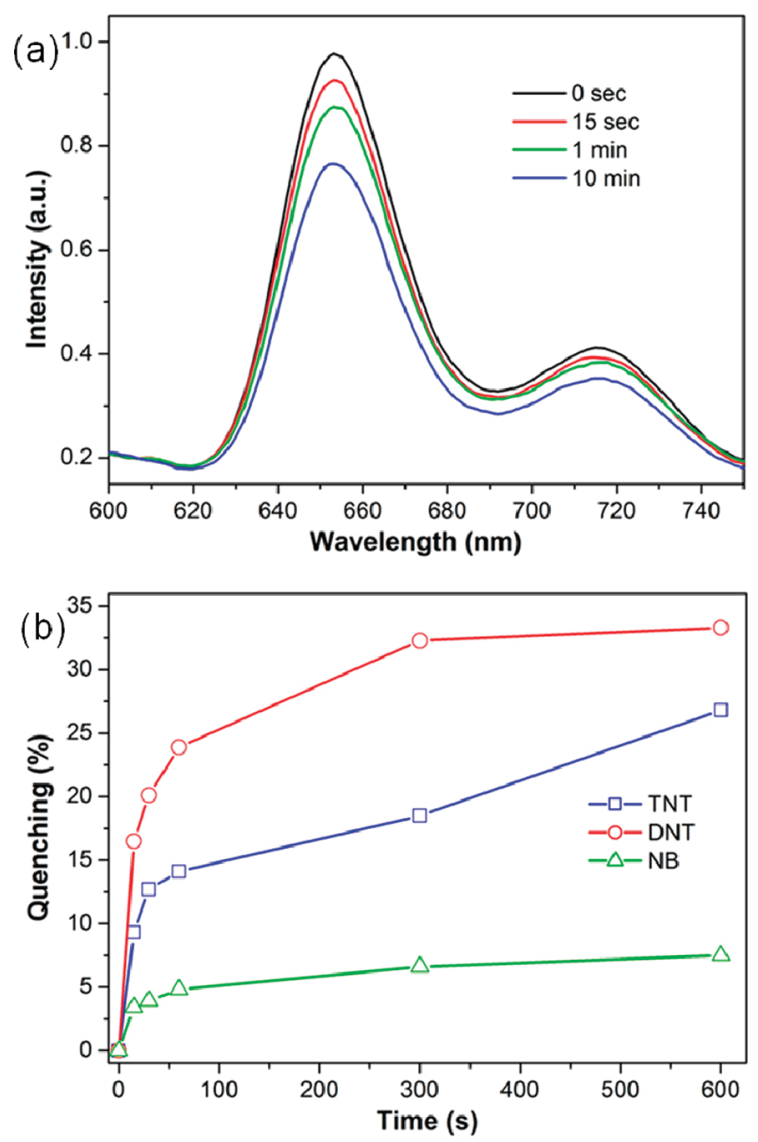

Figure 4. (a) Fluorescence quenching of FSNT surface with TNT. (b) Quenching efficiencies of the sensor upon exposure to TNT, DNT, and NB with respect to time.

xylene, BA, and DBA). Figure 4 shows the fluorescence quenching of the FSNT sensor against TNT exposure on its vapor pressure $(10 \mathrm{ppb})$ at RT. Fluorescence of the sensor was quenched $8.7 \%$ in $15 \mathrm{~s}$ and $29.3 \%$ in $10 \mathrm{~min}$ after exposure to the TNT vapor. The cause of the fluorescence quenching is photoinduced electron transfer between excited $\mathrm{TCPPH}_{2}$ and TNT molecules. When a TNT molecule binds to $\mathrm{TCPPH}_{2}$ dye, the excited electron of the dye molecule was transferred to the LUMO of the TNT molecule rather than relaxation by emitting of light. ${ }^{26}$ The FSNT sensor also exhibited a significantly high fluorescence quenching efficiency after DNT vapor exposure (Figure $4 \mathrm{~b}$ ). The quenching efficiency for DNT was found to be $16.5 \%$ after $15 \mathrm{~s}$ and $33.3 \%$ after $10 \mathrm{~min}$, which is higher than the quenching values obtained for TNT. It is known that the vapor phase fluorescence quenching efficiency of an analyte is mainly determined by its vapor pressure, the exergonicity $\left(-\Phi G^{\circ}\right)$ of electron transfer between dye and analyte, and the binding strength of analyte. The binding constant of TNT to electronrich porphyrin ring is higher than that of DNT because of its extra electron-withdrawing nitro group. ${ }^{34}$ On the other hand, vapor pressure of DNT (180 ppb) is 18-fold higher than the vapor pressure of TNT at RT. As a result of these two competing factors, the sensor exhibited a better quenching efficiency against DNT exposure. In Figure $4 \mathrm{~b}$, fluorescence quenching of the sensor with nitrobenzene (NB) is also presented. NB resulted in a relatively low quenching (7.5\%) after $10 \mathrm{~min}$, compared to TNT and DNT. The binding constant of NB to porphyrin ring is 


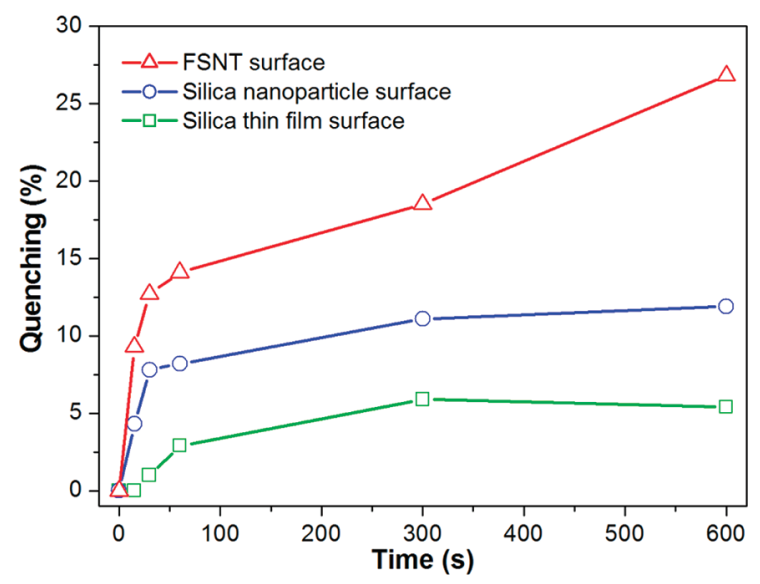

Figure 5. Quenching efficiencies of FNST, silica nanoparticle, and silica thin film sensors upon exposure to TNT with respect to time.

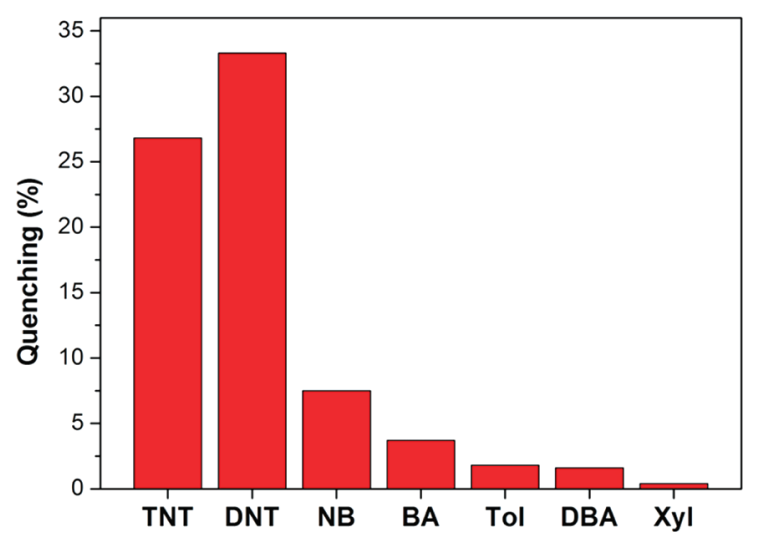

Figure 6. Quenching efficiencies of 7 analytes tested after $10 \mathrm{~min}$ exposure. The FSNT sensor is found to be very selective to the TNT and DNT. NB (nitrobenzene), BA (benzoic acid), Tol (toluene), DBA (dihydroxybenzoic acid), and Xyl (xylene).

expected to be lower than TNT and DNT since NB contains only one electron withdrawing nitro group, and it resulted in low quenching of fluorescence, although NB has relatively higher vapor pressure ( $300 \mathrm{ppm})$ at RT.

The fast response and high quenching efficiency of the FSNT sensor can be attributed to the high surface area of porous FSNT network and also high affinity of the $\mathrm{TCPPH}_{2}$ to the TNT and DNT. Porous structure provides fast diffusion of the analyte molecules, and high surface area enhances analyte-dye interactions. In order to demonstrate the effect of high surface area on fluorescence quenching, we prepared two other $\mathrm{TCPPH}_{2}$ doped control surfaces with lower porosities: a dense silica thin film and a surface composed of silica nanoparticles. The thickness of the silica thin film was determined as $170 \mathrm{~nm}$ by an ellipsometer. The silica nanoparticle sensor surface was investigated by SEM (Figure S4, Supporting Information). The diameters of the nanoparticles were found to be around $60 \mathrm{~nm}$. The nanoparticles were to glass surface without aggregation, and there were some uncoated parts. The fluorescence quenching performances of the control surfaces were tested with TNT (Figure 5). Both silica thin film and nanoparticle surfaces exhibited significantly lower fluorescence quenching (after $10 \mathrm{~min}, 5.4 \%$ and $11.2 \%$, respectively) compared to FSNT surface. The reason of the lower sensitivity of these two sensors is potentially due to their lower surface areas. In addition, it must be noted that nonporous thin film sensor exhibited lower quenching efficiency than slightly porous nanoparticle film.

The selectivity of the sensor was tested with four other control aromatic compounds in addition to TNT, DNT, and NB, and the results are presented in Figure 6. None of these chemicals quenched the fluorescence of the sensor, significantly, even after 10 min of exposure showing the high selectivity of the FSNT sensor against nitro-explosives (e.g., TNT and DNT).

\section{CONCLUSION}

In summary, we studied fluorescence quenching based explosive sensing performance of dye doped peptide nanofiber (ALP) templated high aspect ratio silica nanotubes. A rationally designed peptide nanostructure was used as a template for silica nanotube preparation. The ALP template also catalyzed silica mineralization by the amine groups. To provide explosive sensing property to the silica nanotubes, they were doped with a fluorescent dye. The fluorescent nanotube coated surfaces revealed fast fluorescence quenching against DNT and TNT due to its porous structure. The high selectivity of the FSNT sensor for nitro-explosives was demonstrated by testing the sensor with other control aromatic compounds. The ALP templated high aspect ratio silica nanotubes present a promising platform with their high surface area for sensing and catalysis applications.

\section{ASSOCIATED CONTENT}

S Supporting Information. Additional molecular and structural characterization data. This material is available free of charge via the Internet at http://pubs.acs.org.

\section{AUTHOR INFORMATION}

\section{Corresponding Authors}

*E-mail: moguler@unam.bilkent.edu.tr (M.O.G.); bayindir@ nano.org.tr (M.B.). Telephone: +90 (312) 290 3552. Fax: +90 (312) 2664365 .

\section{ACKNOWLEDGMENT}

We would like to thank M. Guler and Z. Erdogan for their help on measurements and B. Daglar for preparation of silica nanoparticles. This work is supported by TUBITAK under the Grants 109T603, 106G090, and 110M412. M.B. and M.O.G. acknowledge support from the Turkish Academy of Sciences Distinguished Young Scientist Award (TUBA GEBIP).

\section{REFERENCES}

(1) Yang, X.; Tang, H.; Cao, K.; Song, H.; Sheng, W.; Wu, Q. J. Mater. Chem. 2011, 21, 6122.

(2) Fan, R.; Wu, Y.; Li, D.; Yue, M.; Majumdar, A.; Yang, P. J. Am. Chem. Soc. 2003, 125, 5254.

(3) Piao, Y.; Burns, A.; Kim, J.; Wiesner, U.; Hyeon, T. Adv. Funct. Mater. 2008, 18, 3745.

(4) Rao, C. N. R.; Govindaraj, A. Adv. Mater. 2009, 21, 4208.

(5) Liu, Y. H.; Tsai, Y. Y.; Chien, H. J.; Chen, C. Y.; Huang, Y. F.; Chen, J. S.; Wu, Y. C.; Chen, C. C. Nanotechnology 2011, 22, 155102.

(6) Yu, J.; Bai, X.; Suh, J.; Lee, S. B.; Son, S. J. J. Am. Chem. Soc. 2009, $131,15574$. 
(7) Kim, M.; Hong, J.; Lee, J.; Hong, C. K.; Shim, S. E. J. Colloid Interface Sci. 2008, 322, 321.

(8) Tsung-Wu, L.; Hsin-Hui, S. Nanotechnology 2010, 21, 365604.

(9) Chen, Y. J.; Xue, X. Y.; Wang, T. H. Nanotechnology 2005, 16, 1978.

(10) Zhu, J.; Peng, H.; Connor, S. T.; Cui, Y. Small 2009, 5, 437.

(11) Obare, S. O.; Jana, N. R.; Murphy, C. J. Nano Lett. 2001, 1, 601.

(12) Adachi, M.; Harada, T.; Harada, M. Langmuir 1999, 15, 7097.

(13) Adachi, M.; Harada, T.; Harada, M. Langmuir 2000, 16, 2376.

(14) Yuwono, V. M.; Hartgerink, J. D. Langmuir 2007, 23, 5033.

(15) Jung, J. H.; Shinkai, S.; Shimizu, T. Nano Lett. 2002, 2, 17.

(16) Yamanaka, M.; Miyake, Y.; Akita, S.; Nakano, K. Chem. Mater. 2008, 20, 2072.

(17) Xie, C.; Liu, B.; Wang, Z.; Gao, D.; Guan, G.; Zhang, Z. Anal. Chem. 2008, 80, 437.

(18) Acar, H.; Garifullin, R.; Guler, M. O. Langmuir 2011, 27, 1079.

(19) McQuade, D. T.; Pullen, A. E.; Swager, T. M. Chem. Rev. 2000, $100,2537$.

(20) Nie, H.; Zhao, Y.; Zhang, M.; Ma, Y.; Baumgarten, M.; Mullen, K. Chem. Commun. 2011, 47, 1234.

(21) Rose, A.; Zhu, Z.; Madigan, C. F.; Swager, T. M.; Bulovic, V. Nature 2005, 434, 876.

(22) Toal, S. J.; Trogler, W. C. J. Mater. Chem. 2006, 16, 2871.

(23) Long, Y. Y.; Chen, H. B.; Yang, Y.; Wang, H. M.; Yang, Y. F.; Li, N.; Li, K. A.; Pei, J.; Liu, F. Macromolecules 2009, 42, 6501.

(24) Naddo, T.; Che, Y.; Zhang, W.; Balakrishnan, K.; Yang, X.; Yen, M.; Zhao, J.; Moore, J. S.; Zang, L. J. Am. Chem. Soc. 2007, 129, 6978.

(25) Zhang, C.; Che, Y.; Yang, X.; Bunes, B. R.; Zang, L. Chem Commun. 2010, 46, 5560.

(26) Tao, S.; Li, G.; Zhu, H. J. Mater. Chem. 2006, 16, 4521.

(27) Tao, S.; Shi, Z.; Li, G.; Li, P. ChemPhysChem 2006, 7, 1902.

(28) Yildirim, A.; Budunoglu, H.; Deniz, H.; O. Guler, M.; Bayindir, M. ACS Appl. Mater. Interfaces 2010, 2, 2892.

(29) Fang, Q.; Geng, J.; Liu, B.; Gao, D.; Li, F.; Wang, Z.; Guan, G.; Zhang, Z. Chem.-Eur. J. 2009, 15, 11507.

(30) Gao, D.; Wang, Z.; Liu, B.; Ni, L.; Wu, M.; Zhang, Z. Anal. Chem. 2008, 80, 8545.

(31) Stöber, W.; Fink, A.; Bohn, E. J. Colloid Interface Sci. 1968, 26,62 .

(32) Mazor, Y.; Gilead, S.; Benhar, I.; Gazit, E. J. Mol. Biol. 2002, $322,1013$.

(33) Gazit, E. FASEB J. 2002, 16, 77.

(34) Yang, J.-S.; Swager, T. M. J. Am. Chem. Soc. 1998, 120, 11864. 